Anna Kikot

O.I. Komarova, language advisor

Poltava National Technical Yuri Kondratyuk University, Poltava, Ukraine

\title{
The Simulation of Human Intelligence
}

Nowadays, we are extensively feed up with technology of information all around us. Everything we have seen around us is purely a product of high end advancement. The arrival of computers made the importance of information technology rapidly spreading around where everyone has observed its unveiling growth. It's an industry which gathers the procedure of computer hardware, software and networking. Information technology turns as an aide. A standard process that allows great bulks of data to be kept and processed or transmitted at lightning speed. Now, there is more information at hand to make choices, sustain and preserve relations, monitor business activities or track movements. By this, information can be received and acquired at any moment.

Disruptive technologies have become commonplace in the software industry, and lately, artificial intelligence is on many companies' radars.

AI is the simulation of human intelligence processes by machines, especially computer systems. These processes include learning (the acquisition of information and rules for using the information), reasoning (using the rules to reach approximate or definite conclusions), and self-correction.

AI was coined by John McCarthy, an American computer scientist, in 1956 at The Dartmouth Conference where the discipline was born. Today, it is a basic term that encompasses everything from robotic process automation to actual robotics. It has gained prominence recently due, in part, to big data, or the increase in speed, size and variety of data businesses are now collecting. AI can perform tasks such as identifying patterns in the data more efficiently than humans, enabling businesses to gain more insight out of their data.

Artificial intelligence today is properly known as narrow AI (or weak AI), in that it is designed to perform a narrow task (e.g. only facial recognition or only internet searches or only driving a car). However, the long-term goal of many researchers is to create general AI (AGI or strong AI). While narrow AI may outperform humans at whatever its specific task is, like playing chess or solving equations, AGI would outperform humans at nearly every cognitive task.

Most researchers agree that a superintelligent AI is unlikely to exhibit human emotions like love or hate, and that there is no reason to expect AI to become intentionally benevolent or malevolent. Instead, when considering how AI might become a risk, experts think two scenarios most likely:

1. The AI is programmed to do something devastating:

2. The AI is programmed to do something beneficial, but it develops a destructive method for achieving its goal

AI researchers have created many tools to solve the most difficult problems in computer science. Many of their inventions have been adopted by mainstream 
computer science and are no longer considered a part of AI. All of the following were originally developed in AI laboratories: time sharing, interactive interpreters, graphical user interfaces and the computer mouse, rapid development environments, the linked list data structure, automatic storage management, symbolic programming, functional programming, dynamic programming and object-oriented programming.

One of the instances of AI that most people are probably familiar with, video game AI has been used for a very long time-since the very first video games, in fact. But the complexity and effectiveness of that AI has increased exponentially over the past several decades, resulting in video game characters that learn your behaviors, respond to stimuli, and react in unpredictable ways.

Siri, Google Now, and Cortana are all intelligent digital personal assistants on various platforms (iOS, Android, and Windows Mobile). In short, they help find useful information when you ask for it using your voice; you can request for something and the assistant will respond by finding information, relaying information from your phone, or sending commands to other apps.

Many websites now offer customers the opportunity to chat with a customer support representative while they're browsing - but not every site actually has a live person on the other end of the line. In many cases, you're talking to a rudimentary AI. Many of these chat support bots amount to little more than automated responders, but some of them are actually able to extract knowledge from the website and present it to customers when they ask for it.

Many smart home devices now include the ability to learn your behavior patterns and help you save money by adjusting the settings on your thermostat or other appliances in an effort to increase convenience and save energy. For example, turning your oven on when you leave work instead of waiting to get home is a very convenient ability. A thermostat that knows when you're home and adjusts the temperature accordingly can help you save money by not heating the house when you're out.

Lighting is another place where you might see basic artificial intelligence; by setting defaults and preferences, the lights around your house (both inside and outside) might adjust based on where you are and what you're doing; dimmer for watching TV, brighter for cooking, and somewhere in the middle for eating, for example. The uses of AI in smart homes are limited only by our imagination.

Your smartphone, your car, your bank, and your house all use artificial intelligence on a daily basis; sometimes it's obvious what its' doing, like when you ask Siri to get you directions to the nearest gas station. Sometimes it's less obvious, like when you make an abnormal purchase on your credit card and don't get a fraud alert from your bank. AI is everywhere, and it's making a huge difference in our lives every day. 